\title{
OPTIMALITY CRITERIA IN SET-VALUED OPTIMIZATION
}

\author{
C. S. LALITHA, J. DUTTA and MISHA G. GOVIL
}

(Received 24 April 2001; revised 15 May 2002)

Communicated by M. Snedovich

\begin{abstract}
The main aim of this paper is to obtain optimality conditions for a constrained set-valued optimization problem. The concept of Clarke epiderivative is introduced and is used to derive necessary optimality conditions. In order to establish sufficient optimality criteria we introduce a new class of set-valued maps which extends the class of convex set-valued maps and is different from the class of invex set-valued maps.
\end{abstract}

2000 Mathematics subject classification: primary 49J53, 90C 30.

Keywords and phrases: set-valued optimization, contingent epiderivative, Clarke epiderivative, conearcwise connectedness, optimality.

\section{Introduction}

The significance of the study of set-valued maps is due to its applications in various fields such as economics, game theory and differential inclusions (see Aubin and Ekeland [3]). These maps are also involved at various instances in nonsmooth analysis. For example, tangent cones, subgradients and inverse of functions are all set-valued maps.

In the literature various approaches have been followed in defining the concept of derivative for set-valued maps. Aubin [1,2] introduced the concept of derivatives in terms of the contingent cones and the Clarke [5] tangent cones, to the graph of set-valued maps. Luc [10] and Corley [6] established optimality criteria in terms of these derivatives for set-valued optimization problems. However, while characterizing optimality conditions, it is useful to consider derivatives involving epigraph of setvalued maps rather than their graphs (see [9, 11]). Jahn and Rauh [9] introduced the notion of epiderivative via contingent cones to the epigraph of set-valued maps.

(C) 2003 Australian Mathematical Society $1446-7887 / 03 \$ \mathrm{~A} 2.00+0.00$ 
It is important to note that Jahn and Rauh [9] introduced a single-valued map as the derivative of a set-valued map. Since the contingent cones are not necessarily convex, Gotz and Jahn [8] required the convexity of the set-valued maps in order to derive the necessary optimality conditions for a set-valued optimization problem. Sach and Craven [11] introduced a derivative, in the sense of Aubin and Ekeland [3], by considering Clarke tangent cone to the epigraph where the derivative introduced is a set-valued map. The notion of invexity for set-valued maps was also introduced by Sach and Craven [11] and optimality conditions were obtained for a constrained set-valued optimization problem. Motivated by the works of Gotz and Jahn [8] and Sach and Craven [11] we introduce here the notion of a Clarke epiderivative of a set-valued map. We would also like to mention that this Clarke epiderivative is a single-valued map, unlike the derivative considered by Sach and Craven [11].

In Section 2 some basic definitions and results are given. In Section 3 we introduce the notion of Clarke epiderivative for set-valued maps in terms of Clarke tangent cone. Fritz John type necessary optimality conditions are obtained in this section, for a constrained set-valued optimization problem in terms of the Clarke epiderivative. In Section 4 of the paper the notion of arcwise connectedness is introduced for set-valued maps as an extension of the notion of convexity. We demonstrate the effectiveness of this new class of maps in proving sufficient optimality criteria for the optimization problem considered in Section 3. With the help of an example it has been shown that this class of set-valued maps is different from the class of invex set-valued maps considered by Sach and Craven [11].

\section{Preliminaries}

For any real normed linear space $Y$, let $0_{Y}$ denote the origin of $Y$ and let $Y^{*}$ denote the dual space of $Y$. Let $C$ be a pointed closed convex cone in $Y$ and let

$$
C^{*}=\left\{\varphi \in Y^{*}: \varphi(y) \geq 0 \forall y \in C\right\}
$$

be the dual cone for $C$. For $A \subseteq Y$, define the contingent cone $T\left(A, y^{*}\right)$ to $A$ at $y^{*}$ as

$$
T\left(A, y^{*}\right)=\left\{h \in Y: \exists t_{n} \downarrow 0, \exists h_{n} \rightarrow h \text { with } y^{*}+t_{n} h_{n} \in A \quad \forall n\right\}
$$

and the normal cone $N\left(A, y^{*}\right)$ to $A$ at $y^{*}$ as

$$
N\left(A, y^{*}\right)=\left\{\varphi \in Y^{*}: \varphi(y) \leq 0 \forall y \in T\left(A, y^{*}\right)\right\} .
$$

The Clarke tangent cone $T_{c}\left(A, y^{*}\right)$ to $A$ at $y^{*}$ is given as

$$
T_{c}\left(A, y^{*}\right)=\left\{h \in Y: \forall y_{n} \rightarrow y^{*} \text {, and } \forall t_{n} \downarrow 0, \exists h_{n} \rightarrow h \text { with } y_{n}+t_{n} h_{n} \in A \forall n\right\} \text {. }
$$


The Clarke tangent cone $T_{c}\left(A, y^{*}\right)$ is a closed convex cone and $T_{c}\left(A, y^{*}\right) \subseteq T\left(A, y^{*}\right)$. If $A$ is a convex set then $T_{c}\left(A, y^{*}\right)=T\left(A, y^{*}\right)$.

We now present the concept of contingent epiderivative introduced by Jahn and Rauh [9] for set-valued maps. Let $X$ be a real normed linear space and $S$ be a nonempty subset of $X$. Let $F: S \rightarrow 2^{Y}$ be a set-valued map. The epigraph of $F$, denoted by epi $(F)$, is defined as

$$
\operatorname{epi}(F)=\{(x, y) \in X \times Y: x \in S, y \in F(x)+C\} .
$$

Let $x^{*} \in S$ with $y^{*} \in F\left(x^{*}\right)$. A single valued map $D F\left(x^{*}, y^{*}\right): X \rightarrow Y$ whose epigraph equals the contingent cone to the epigraph of $F$ at $\left(x^{*}, y^{*}\right)$, that is, epi $\left(D F\left(x^{*}, y^{*}\right)\right)=T\left(\operatorname{epi}(F),\left(x^{*}, y^{*}\right)\right)$ is called the contingent epiderivative of $F$ at $\left(x^{*}, y^{*}\right)$. The map $D F\left(x^{*}, y^{*}\right)$ is not necessarily convex as $T\left(\right.$ epi $\left.(F),\left(x^{*}, y^{*}\right)\right)$ may not be convex.

Sach and Craven [11] introduced the concept of derivative, in the sense of Aubin and Ekeland [3], for $\hat{F}(x)=F(x)+C$. Let $\operatorname{gr}(F)$ denote the graph of a set-valued map $F: X \rightarrow 2^{Y}$, given by

$$
\operatorname{gr}(F)=\{(x, y) \in X \times Y: x \in X, y \in F(x)\} .
$$

The derivative of $\hat{F}$ at $\left(x^{*}, y^{*}\right) \in g r(F)$ is the set-valued map $D \hat{F}\left(x^{*}, y^{*}\right)$ whose graph is $T_{c}\left(\right.$ epi $\left.(F),\left(x^{*}, y^{*}\right)\right)$, that is $\operatorname{gr}\left(D \hat{F}\left(x^{*}, y^{*}\right)\right)=T_{c}\left(\right.$ epi $\left.(F),\left(x^{*}, y^{*}\right)\right)$.

A set-valued map $F: X \rightarrow 2^{Y}$ is said to be $C$-convex on $S$, where $S$ is a convex subset of $X$, if for all $x_{1}, x_{2} \in S, \alpha \in[0,1]$

$$
(1-\alpha) F\left(x_{1}\right)+\alpha F\left(x_{2}\right) \subseteq F\left((1-\alpha) x_{1}+\alpha x_{2}\right)+C .
$$

The following alternative theorem is by Craven [7, Theorem 3.4.2].

LEMMA 2.1. Let $X$ and $Y$ be real normed linear spaces and $C$ be a closed convex cone in $Y$ with nonempty interior. Let the single valued map $f: S \rightarrow Y$ be $C$-convex where $S$ is a convex subset of $X$. Then exactly one of the following systems has a solution

(i) There exists $x \in S$ such that $-f(x) \in$ int $C$.

(ii) There exists $\varphi \in C^{*}, \quad \varphi \neq 0_{Y^{*}}$, such that $\varphi(f(x)) \geq 0$ for all $x \in S$.

\section{Necessary optimality criteria}

In this section we first introduce the concept of Clarke epiderivative for a set-valued $\operatorname{map} F: X \rightarrow 2^{Y}$. 
Definition 3.1. Let $x^{*} \in X$ with $y^{*} \in F\left(x^{*}\right)$. A single valued map $D_{c} F\left(x^{*}, y^{*}\right)$ : $X \rightarrow Y$ whose epigraph equals the Clarke tangent cone to the epigraph of $F$ at $\left(x^{*}, y^{*}\right)$, that is, epi $\left(D_{c} F\left(x^{*}, y^{*}\right)\right)=T_{c}\left(\operatorname{epi}(F),\left(x^{*}, y^{*}\right)\right)$ is called the Clarke epiderivative of $F$ at $\left(x^{*}, y^{*}\right)$.

As $T_{c}$ (epi $\left.(F),\left(x^{*}, y^{*}\right)\right)$ is a convex set it follows that $D_{c} F\left(x^{*}, y^{*}\right): X \rightarrow Y$ is a $C$-convex map, that is, for all $x_{1}, x_{2} \in X, \alpha \in[0,1]$

$$
(1-\alpha) D_{c} F\left(x^{*}, y^{*}\right)\left(x_{1}\right)+\alpha D_{c} F\left(x^{*}, y^{*}\right)\left(x_{2}\right) \in D_{c} F\left(x^{*}, y^{*}\right)\left((1-\alpha) x_{1}+\alpha x_{2}\right)+C .
$$

On the lines of Theorem 4 of Jahn and Rauh [9] we can establish the following result.

LEMMA 3.1. Let $F: X \rightarrow 2^{Y}$ be a set-valued map and $C$ be a pointed convex cone in $Y$. If the Clarke epiderivative $D_{c} F\left(x^{*}, y^{*}\right)$ exists, then it is sublinear.

REMARK 3.1. It can be seen that if both the contingent epiderivative $D F\left(x^{*}, y^{*}\right)$ and the Clarke epiderivative $D_{c} F\left(x^{*}, y^{*}\right)$ exist, then for all $x \in X$

$$
D_{c} F\left(x^{*}, y^{*}\right)(x) \in D F\left(x^{*}, y^{*}\right)(x)+C
$$

as $T_{c}\left(\operatorname{epi}(F),\left(x^{*}, y^{*}\right)\right) \subseteq T\left(\operatorname{epi}(F),\left(x^{*}, y^{*}\right)\right)$.

REMARK 3.2. On comparing the definitions of the Clarke epiderivative and the derivative introduced by Sach and Craven [11] it follows that

$$
\operatorname{gr}\left(D \hat{F}\left(x^{*}, y^{*}\right)\right)=\operatorname{epi}\left(D_{c} F\left(x^{*}, y^{*}\right)\right),
$$

that is, $D \hat{F}\left(x^{*}, y^{*}\right)=D_{c} F\left(x^{*}, y^{*}\right)+C$.

The Clarke epiderivative is a single valued map whereas the derivative $D \hat{F}\left(x^{*}, y^{*}\right)$ is a set-valued map. Therefore, it is meaningful to introduce the Clarke epiderivative $D_{c} F\left(x^{*}, y^{*}\right)$ as it is easier to deal with a single valued map rather than a set-valued map.

We now derive the necessary optimality criteria for a constrained set-valued optimization problem. Let $X, Y, Z$ be real normed linear spaces and let $C$ and $D$ be pointed convex cones in $Y$ and $Z$ respectively, with nonempty interiors. Let $F: X \rightarrow 2^{Y}$ and $G: X \rightarrow 2^{Z}$ be set-valued maps. Consider the following set-valued optimization problem

W Min $F(x)$ subject to $G(x) \cap(-D) \neq \phi$.

Let $A=\{x \in X: G(x) \cap(-D) \neq \phi\}$. We assume throughout that $F(x) \neq \phi$, $G(x) \neq \phi$, for every $x \in X$. A point $\left(x^{*}, y^{*}, z^{*}\right)$ is said to be a weak minimizer 
of $(P)$ if $x^{*} \in A, y^{*} \in F\left(x^{*}\right), z^{*} \in G\left(x^{*}\right) \cap(-D)$ and $\left(y^{*}-F(A)\right) \cap$ int $C=\phi$, where $F(A)=\bigcup_{x \in A} F(x)$. The set-valued map $(F, G): X \rightarrow 2^{Y \times Z}$ is defined as $(F, G)(x)=F(x) \times G(x)$ for all $x \in X$. We now establish the following necessary optimality theorem for Problem $(P)$.

THEOREM 3.1. If $\left(x^{*}, y^{*}, z^{*}\right)$ is a weak minimizer of $(P)$, then there exists

$$
\left.(\varphi, \psi) \in C^{*} \times D^{*} \backslash\left(0_{Y^{*}}, 0_{Z^{*}}\right)\right\}
$$

such that for all $(y, z) \in D_{c}(F, G)\left(x^{*}, y^{*}, z^{*}\right)(X), \varphi(y)+\psi(z) \geq 0$ and $\psi\left(z^{*}\right)=0$.

PROOF. We will first prove that

$$
\left[D_{c}(F, G)\left(x^{*}, y^{*}, z^{*}\right)(x)+\left(0_{Y}, z^{*}\right)\right] \notin(- \text { int } C) \times(- \text { int } D)
$$

for any $x \in X$, where int $C$ denotes the interior of $C$. Suppose on the contrary there exists $u \in X$ such that

$$
(v, w)+\left(0_{Y}, z^{*}\right) \in(-\operatorname{int} C) \times(- \text { int } D),
$$

where $(v, w)=D_{c}(F, G)\left(x^{*}, y^{*}, z^{*}\right)(u)$. By the definition, we get

$$
(u, v, w) \in \operatorname{epi}\left(D_{c}(F, G),\left(x^{*}, y^{*}, z^{*}\right)\right)=T_{c}\left(\operatorname{epi}(F, G),\left(x^{*}, y^{*}, z^{*}\right)\right) .
$$

As $T_{c}$ (epi $\left.(F, G),\left(x^{*}, y^{*}, z^{*}\right)\right) \subseteq T\left(\operatorname{epi}(F, G),\left(x^{*}, y^{*}, z^{*}\right)\right)$ there exists a sequence $\left(x_{n}, y_{n}, z_{n}\right)$ with $x_{n} \in X, y_{n} \in F\left(x_{n}\right)+C, z_{n} \in G\left(x_{n}\right)+D$ and a sequence $\left(\lambda_{n}\right)$ of positive real numbers such that $\lim _{n \rightarrow \infty}\left(x_{n}, y_{n}, z_{n}\right)=\left(x^{*}, y^{*}, z^{*}\right)$ and

$$
\lim _{n \rightarrow \infty} \lambda_{n}\left(x_{n}-x^{*}, y_{n}-y^{*}, z_{n}-z^{*}\right)=(u, v, w) .
$$

Since $v \in-$ int $C$ and $w+z^{*} \in-$ int $D$, there exist natural numbers $N_{1}, N_{2}$ such that $\lambda_{n}\left(y_{n}-y^{*}\right) \in-$ int $C \forall n \geq N_{1}$ and $\lambda_{n}\left(z_{n}-z^{*}\right)+z^{*} \in-$ int $D \forall n \geq N_{2}$. Choose $N \geq \max \left(N_{1}, N_{2}\right)$. Then

$$
y_{N}-y^{*} \in-\text { int } C, \quad z_{N}-\left(1-1 / \lambda_{N}\right) z^{*} \in-\text { int } D .
$$

Thus, we have that $y^{*} \in y_{N}+$ int $C$. As $y_{N} \in F\left(x_{N}\right)+C$, it follows from (1) that $y^{*} \in F\left(x_{N}\right)+C+$ int $C$, that is,

$$
\left(y^{*}-F\left(x_{N}\right)\right) \cap \operatorname{int} C \neq \phi .
$$

We can suitably choose $N$ such that $\lambda_{N}>1$. Since $z^{*} \in-D$ we have $\left(1-\left(1 / \lambda_{N}\right)\right) z^{*} \in$ $-D$. As $D$ is a convex cone, on using (1) it follows that $z_{N} \in-$ int $D$. Also since $z_{N} \in G\left(x_{N}\right)+D$ we have $z_{N}=\alpha+\beta$ where $\alpha \in G\left(x_{N}\right), \beta \in D$. As $z_{N} \in-$ int $D$, 
we have $\alpha \in-\beta-$ int $D \subseteq-D-$ int $D \subseteq-D$ and hence $\alpha \in G\left(x_{N}\right) \cap(-D)$ that is, $x_{N}$ is a feasible solution of $(P)$. Thus (2) contradicts the fact that $\left(x^{*}, y^{*}, z^{*}\right)$ is a weak minimizer of $(P)$.

Since $D_{c}(F, G)\left(x^{*}, y^{*}, z^{*}\right)$ is sublinear (see Lemma 3.1) it is a $C \times D$-convex map and hence by Lemma 2.1 there exists $(\varphi, \psi) \in C^{*} \times D^{*} \backslash\left\{\left(0_{Y^{*}}, 0_{Z^{*}}\right)\right\}$ such that

$$
\varphi(y)+\psi\left(z+z^{*}\right) \geq 0
$$

for all $(y, z) \in D_{c}(F, G)\left(x^{*}, y^{*}, z^{*}\right)(X)$. As $D_{c}(F, G)\left(x^{*}, y^{*}, z^{*}\right)$ is positively homogeneous, we have $D_{c}(F, G)\left(x^{*}, y^{*}, z^{*}\right)(0)=\left(0_{Y}, 0_{Z}\right)$. Thus from (3) it follows that $\psi\left(z^{*}\right) \geq 0$. Also as $z^{*} \in-D$, we have $\psi\left(z^{*}\right) \leq 0$ and hence $\psi\left(z^{*}\right)=0$. Since $\psi$ is a continuous linear function and $\psi\left(z^{*}\right)=0$ it follows from (3) that $\varphi(y)+\psi(z) \geq 0$ for all $(y, z) \in D_{c}(F, G)\left(x^{*}, y^{*}, z^{*}\right)(X)$.

REMARK 3.3. In a recent paper by Gotz and Jahn [8] necessary optimality conditions were obtained for Problem $(P)$ in terms of the contingent epiderivative assuming the cone-convexity of the set-valued maps $F$ and $G$. However when the necessary optimality conditions are derived in terms of the Clarke epiderivative no convexity assumption is required on the set-valued maps $F$ and $G$.

REMARK 3.4. Sach and Craven [11, Theorem 1] obtained the Fritz-John necessary optimality conditions in terms of the derivative of the set-valued map $\left(F(x)-y^{*}\right) \times$ $G(x)+(C \times D)$. However it may be noted that in Theorem 3.1 we obtain an additional complementarity condition $\psi\left(z^{*}\right)=0$ unlike the Fritz-John optimality conditions of Sach and Craven [11].

\section{Sufficient optimality criteria}

In this section we first introduce a new class of set-valued maps which extends the class of convex set-valued maps and use it to derive sufficient optimality conditions for the optimization problem $(P)$.

Avriel [4] introduced the concept of arcwise connectedness as a generalization of convexity by replacing the line segment joining two points by a continuous arc. A subset $S$ of $X$ is said to be an arcwise connected set if for all $x_{1}, x_{2} \in S$ there exists a continuous arc $H_{x_{1}, x_{2}}(\alpha)$ defined on $[0,1]$ with a value in $S$ such that $H_{x_{1}, x_{2}}(0)=x_{1}$ and $H_{x_{1}, x_{2}}(1)=x_{2}$.

DEFINITION 4.1. A set-valued map $F: X \rightarrow 2^{Y}$ is said to be $C$-arcwise connected at $x^{*} \in S$, where $S$ is an arcwise connected subset of $X$ and $C$ is a pointed convex cone in $Y$, if for all $x \in S, \alpha \in[0,1]$,

$$
(1-\alpha) F\left(x^{*}\right)+\alpha F(x) \subseteq F\left(H_{x^{*}, x}(\alpha)\right)+C .
$$


A set-valued map $F$ is said to be $C$-arcwise connected on $S$, if it is $C$-arcwise connected at each $x^{*} \in S$.

Clearly every $C$-convex set-valued map is $C$-arcwise connected where $H_{x_{1}, x_{2}}(\alpha)=$ $(1-\alpha) x_{1}+\alpha x_{2}$. The following examples illustrate that the converse is not necessarily true.

EXAMPLE 4.1. Let $X=R^{2}, Y=R, C=R_{+}$and

$$
S=\left\{\left(x_{1}, x_{2}\right): x_{1}^{2}+x_{2}^{2} \geq 1, x_{1} \geq 0, x_{2} \geq 0\right\} .
$$

Define $H_{x, u}(\alpha)=\left(\left((1-\alpha) x_{1}^{2}+\alpha u_{1}^{2}\right)^{1 / 2},\left((1-\alpha) x_{2}^{2}+\alpha u_{2}^{2}\right)^{1 / 2}\right)$, where $x=\left(x_{1}, x_{2}\right)$ and $u=\left(u_{1}, u_{2}\right)$. Clearly $S$ is an arcwise connected set. Define $F: X \rightarrow 2^{Y}$ as

$$
F(x)= \begin{cases}{[0,2]} & \text { if } x_{1}^{2}+x_{2}^{2} \geq 1 \\ {[3,5[} & \text { if } x_{1}^{2}+x_{2}^{2}<1\end{cases}
$$

The set-valued map $F$ is $C$-arcwise connected on $S$, but it is not $C$-convex because for $x=(1,0), u=(0,1), \alpha=1 / 2$ the following does not hold

$$
(1-\alpha) F(x)+\alpha F(u) \subseteq F((1-\alpha) x+\alpha u)+C .
$$

EXAMPLE 4.2. Let $X=R, Y$ be the set of real sequences converging to zero, $C$ be the set of nonnegative real sequences converging to zero and $S=R_{+} . \quad Y$ is a normed linear space with $\|y\|=\sup _{n}\left|y_{n}\right|$ for $y=\left(y_{n}\right) \in Y$. Define $H_{x, u}(\alpha)=$ $(1-\alpha)^{1 / 2} x+\alpha^{1 / 2} u$, for $x, u \in S$. Define $F: R \rightarrow 2^{Y}$ as

$$
F(x)=\left\{\left\{x^{2} / n\right\},\left\{-x^{2} / n\right\}\right\} .
$$

From the definition of $F$ we can see that $F$ is set-valued map since at each $x \in R$, $F(x)$ is the set consisting of two sequences from $Y$, defined in that particular fashion. It can be seen that $F$ is a $C$-arcwise connected set-valued map on $S$, but it is not $C$-convex, because for $x=1, u=3, \alpha=1 / 2$ the following does not hold

$$
(1-\alpha) F(x)+\alpha F(u) \subseteq F((1-\alpha) x+\alpha u)+C .
$$

Sach and Craven [11] generalized the concept of invexity for set-valued maps. One of the generalizations, namely invex 2 is given in terms of the the derivative $D \hat{F}\left(x^{*}, y^{*}\right)$ of $\hat{F}$ at $\left(x^{*}, y^{*}\right)$ where $\hat{F}(x)=F(x)+C$. A set-valued map $F: X \rightarrow 2^{Y}$ is said to be invex 2 at $\left(x^{*}, y^{*}\right) \in \operatorname{gr}(F)$, if for every $x \in X$ there exists $\eta \in X$ such that

$$
F(x)+C-y^{*} \subseteq D \hat{F}\left(x^{*}, y^{*}\right)(\eta)
$$

We now give an example of a $C$-arcwise connected set-valued valued map which is not invex 2 for any $\eta \in X$. 
EXAMPLE 4.3. Let $X=Y=R, S=C=R_{+}$. Define $H_{x^{*}, x}(\alpha)=\left((1-\alpha) x^{*^{2}}+\right.$ $\left.\alpha x^{2}\right)^{1 / 2}$ for $x^{*}, x \in S$. Define $F: X \rightarrow 2^{Y}$ as $F(x)=\left[-x^{2}, 0\right]$. It can be seen that $F$ is $C$-arcwise connected on $S$ but is not invex 2 at $\left(x^{*}, y^{*}\right)=(0,0)$, because for $(x, y)=(1,-1)$ the following does not hold

$$
F(x)-y^{*}+C \subseteq D \hat{F}\left(x^{*}, y^{*}\right)(\eta)
$$

for any $\eta \in X$.

We now characterize a $C$-arcwise connected set-valued map in terms of its epigraph.

DEFINITION 4.2. A subset $A \times B$ of $X \times Y$ is said to be arcwise-convex if $A$ is an arcwise connected subset of $X$ and $B$ is a convex subset of $Y$.

THEOREM 4.1. Let $F: S \rightarrow 2^{Y}$ be a set-valued map, where $S$ is an arcwise connected set. The set-valued map $F$ is $C$-arcwise connected, if and only if, epi $(F)$ is an arcwise-convex subset of $X \times Y$.

Proof. Let $F$ be a $C$-arcwise connected set-valued map. Let $\left(x_{i}, y_{i}\right) \in \operatorname{epi}(F)$, $i=1,2$. By definition for $\alpha \in[0,1]$, we have

$$
(1-\alpha) F\left(x_{1}\right)+\alpha F\left(x_{2}\right) \subseteq F\left(H_{x_{1}, x_{2}}(\alpha)\right)+C .
$$

Since $(1-\alpha) y_{1}+\alpha y_{2} \in(1-\alpha) F\left(x_{1}\right)+\alpha F\left(x_{2}\right)+C$, we have

$$
(1-\alpha) y_{1}+\alpha y_{2} \in F\left(H_{x_{1}, x_{2}}(\alpha)\right)+C .
$$

Thus for $\alpha \in[0,1],\left(H_{x_{1}, x_{2}}(\alpha),(1-\alpha) y_{1}+\alpha y_{2}\right) \in \operatorname{epi}(F)$.

Conversely, let $x_{i} \in S, y_{i} \in F\left(x_{i}\right), i=1,2$. Clearly $\left(x_{i}, y_{i}\right) \in \operatorname{epi}(F), i=1,2$. As epi $(F)$ is an arcwise-convex set, for $\alpha \in[0,1]$ we have

$$
\left(H_{x_{1}, x_{2}}(\alpha),(1-\alpha) y_{1}+\alpha y_{2}\right) \in \operatorname{epi}(F),
$$

that is, for $\alpha \in[0,1],(1-\alpha) y_{1}+\alpha y_{2} \in F\left(H_{x_{1}, x_{2}}(\alpha)\right)+C$. Hence $F$ is a $C$-arcwise connected set-valued map on $S$.

The following theorems give necessary conditions for $C$-arcwise connectedness of a set-valued map.

THEOREM 4.2. If $F: X \rightarrow 2^{Y}$ is a $C$-arcwise connected set-valued map on $S$, where $S$ is an arcwise connected subset of $X$, then for all $x^{*}, x \in S, y^{*} \in F\left(x^{*}\right)$

$$
F(x)-y^{*} \subseteq D F\left(x^{*}, y^{*}\right)\left(H_{x^{*}, x}^{\prime}(0+)\right)+C
$$

where

$$
H_{x^{*}, x}^{\prime}(0+)=\lim _{\alpha \downarrow 0} \frac{H_{x^{*}, x}(\alpha)-H_{x^{*}, x}(0)}{\alpha} .
$$


We assume that $H_{x^{*}, x}^{\prime}(0+)$ exists for all $x^{*}, x \in S$.

PROOF. Let $x \in S$ and $y \in F(x)$. Define a sequence $\left(x_{n}, y_{n}\right)$ as

$$
x_{n}=H_{x^{*}, x}(1 / n), \quad y_{n}=y^{*}+\left(y-y^{*}\right) / n .
$$

As $S$ is an arcwise connected set, it follows that $x_{n} \in S$. Also $\lim _{n \rightarrow \infty} y_{n}=y^{*}$ and $\lim _{n \rightarrow \infty} x_{n}=x^{*}$. As $F$ is a $C$-arcwise connected set-valued map, we have

$$
y_{n} \in\left(1-\frac{1}{n}\right) F\left(x^{*}\right)+\frac{1}{n} F(x) \subseteq F\left(H_{x^{*}, x}\left(\frac{1}{n}\right)\right)+C,
$$

that is, $y_{n} \in F\left(x_{n}\right)+C$. Hence the elements of the sequence $\left(x_{n}, y_{n}\right)$ belong to epi $(F)$ with $\lim _{n \rightarrow \infty}\left(x_{n}, y_{n}\right)=\left(x^{*}, y^{*}\right)$. Moreover,

$$
\lim _{n \rightarrow \infty} n\left(x_{n}-x^{*}\right)=\lim _{n \rightarrow \infty} n\left(H_{x^{*}, x}(1 / n)-H_{x^{*}, x}(0)\right)=H_{x^{*}, x}^{\prime}(0+) .
$$

Hence $\lim _{n \rightarrow \infty} n\left(x_{n}-x^{*}, y_{n}-y^{*}\right)=\left(H_{x^{*}, x}^{\prime}(0+), y-y^{*}\right)$ and consequently

$$
\left(H_{x^{*}, x}^{\prime}(0+), y-y^{*}\right) \in T\left(\operatorname{epi}(F),\left(x^{*}, y^{*}\right)\right)=\operatorname{epi}\left(D F\left(x^{*}, y^{*}\right)\right) .
$$

Therefore, $y-y^{*} \in D F\left(x^{*}, y^{*}\right)\left(H_{x^{*}, x}^{\prime}(0+)\right)+C$ and hence the result.

THEOREM 4.3. Let $F: X \rightarrow 2^{Y}$ be a $C$-arcwise connected set-valued map on $S$, where $S$ is an arcwise connected subset of $X$. Assume that $H_{x^{\cdot}, x}^{\prime}(0+)$ (as defined in Theorem 4.2) exists for all $x^{*}, x \in S$ then for all $(\varphi,-\psi) \in N\left(\operatorname{epi}(F),\left(x^{*}, y^{*}\right)\right)$, $x^{*} \in S, y^{*} \in F\left(x^{*}\right), \psi \in C^{*},\|\psi\|=1$ and all $x \in S$

$$
\sigma_{F(x)}(\psi)-\psi\left(y^{*}\right) \geq \varphi\left(H_{x^{*}, x}^{\prime}(0+)\right)
$$

where $\sigma_{F(x)}(\psi)=\inf _{y \in F(x)} \psi(y)$.

Proof. By the previous theorem for any $y \in F(x)$, we have

$$
y-\mathbf{y}^{*} \in D F\left(x^{*}, y^{*}\right)\left(H_{x^{*}, x}^{\prime}(0+)\right)+C,
$$

that is,

$$
\left(H_{x^{*}, x}^{\prime}(0+), y-y^{*}\right) \in \operatorname{epi}\left(D F\left(x^{*}, y^{*}\right)\right)=T\left(\operatorname{epi}(F),\left(x^{*}, y^{*}\right)\right) .
$$

As $(\varphi,-\psi) \in N\left(\operatorname{epi}(F),\left(x^{*}, y^{*}\right)\right)$ we have $\varphi\left(H_{x^{*}, x}^{\prime}(0+)\right)-\psi\left(y-y^{*}\right) \leq 0$ which implies the result.

We now turn to the main result of this section. Sufficient optimality conditions are obtained in terms of contingent epiderivative and not in terms of Clarke epiderivative, as necessary condition for $C$-arcwise connectedness set-valued map is known in terms of contingent epiderivative (see Theorem 4.2.). 
THEOREM 4.4. Let $(F, G): X \rightarrow 2^{Y} \times 2^{Z}$ be a $(C \times D)$-arcwise connected setvalued map on $S$, where $A=\{x \in X: G(x) \cap(-D) \neq \phi\} \subseteq S$ and $S$ is an arcwise connected subset of $X$. If $x^{*} \in A, y^{*} \in F\left(x^{*}\right), z^{*} \in G\left(x^{*}\right) \cap(-D),(\varphi, \psi) \in$ $\left(C^{*}, D^{*}\right) \backslash\left\{\left(0_{Y}^{*}, 0_{Z}^{*}\right)\right\}$ are such that

$$
\varphi(y)+\psi(z) \geq 0
$$

and

$$
\psi\left(z^{*}\right)=0 .
$$

for all $(y, z) \in D(F, G)\left(x^{*}, y^{*}, z^{*}\right)(X)$, then $\left(x^{*}, y^{*}, z^{*}\right)$ is a weak minimizer of $(P)$.

PROOF. On the contrary let $\left(x^{*}, y^{*}, z^{*}\right)$ be not a weak minimizer of $(P)$. Then there exist $x \in A, y \in F(x), z \in G(x) \cap(-D)$ such that $y^{*}-y \in$ int $C$. This implies that

$$
\varphi\left(y-y^{*}\right)<0, \quad \psi(z) \leq 0 .
$$

As $(F, G)$ is a $(C \times D)$-arcwise connected set-valued map on $S$, it follows from Theorem 4.2 that $(y, z)-\left(y^{*}, z^{*}\right) \in D(F, G)\left(x^{*}, y^{*}, z^{*}\right)\left(H_{x^{*}, x}^{\prime}(0+)\right)+(C \times D)$. Hence for some $\left(y^{0}, z^{0}\right) \in D(F, G)\left(x^{*}, y^{*}, z^{*}\right)\left(H_{x^{*}, x}^{\prime}(0+)\right),\left(c^{0}, d^{0}\right) \in C \times D$ we have

$$
(y, z)-\left(y^{*}, z^{*}\right)=\left(y^{0}, z^{0}\right)+\left(c^{0}, d^{0}\right) .
$$

As $\left(c^{0}, d^{0}\right) \in C \times D$ we get

$$
\varphi\left(c^{0}\right) \geq 0, \quad \psi\left(d^{0}\right) \geq 0 .
$$

Using (5), (6), and (8) in (7) we get

$$
\varphi\left(y^{0}\right)+\psi\left(z^{0}\right)<0
$$

which contradicts (4) and hence we arrive at the result.

\section{Acknowledgement}

The authors are grateful to Professor B. D. Craven for his helpful comments which have vastly improved the earlier version of the paper.

\section{References}

[1] J.-P. Aubin, 'Contingent derivatives of set-valued maps and existence of solutions to nonlinear inclusions and differential inclusions', in: Mathematical analysis and applications. Part A (ed. I. Nachbin), Adv. in Math. Suppl. Stud. 7a (Academic Press, New York, 1981) pp. 159-229. 
[2] — - 'Ioffe's fans and generalized derivatives of vector valued maps', in: Convex analysis and optimization (London, 1980) (eds. J.-P. Aubin and R. Vinter), Research Notes in Math. 57 (Pitman, Boston, 1982) pp. 1-17.

[3] J.-P. Aubin and I. Ekeland, Applied nonlinear analysis (Wiley, New York, 1984).

[4] M. Avriel, Nonlinear programming: theory and method (Prentice-Hall, Englewood Cliffs, New Jersey, 1976).

[5] F. H. Clarke, Optimization and nonsmooth analysis (John Wiley, New York, 1983).

[6] H. W. Corley, 'Optimality conditions for maximizations of set-valued functions', J. Optim. Theory Appl. 58 (1988), 1-10.

[7] B. D. Craven, Control and optimization (Chapman and Hall Mathematics, 1995).

[8] A. Gotz and J. Jahn, 'The Lagrange multiplier rule in set-valued optimization', SIAM J. Optim. 10 (1999), 331-344.

[9] J. Jahn and R. Rauh, 'Contingent epiderivative and set-valued optimization', Math. Methods Oper. Res. 46 (1997), 193-211.

[10] D. T. Luc, 'Contingent derivatives of set-valued maps and applications to vector optimization', Math. Programming 50 (1991), 99-111.

[11] P. H. Sach and B. D. Craven, 'Invexity in multifunction optimization', Numer. Funct. Anal. Optim. 12 (1991), 383-394.

Department of Mathematics

Rajdhani College

Raja Garden

New Delhi-110015

India

Department of Mathematics

Shri Ram College of Commerce

University of Delhi

Delhi-110007

India
Department of Mathematics India Institute of Technology

Kanpur-208016

India

e-mail: jdutta@iitk.ac.in 
J. Aust. Math. Soc. 75 (2003) 